\title{
Polynomial Chaos Based Variability Analysis of Multiport Systems
}

\author{
D. Spina*, F. Ferranti*, T. Dhaene*, L. Knockaert*, G. Antonini ${ }^{+}$ \\ *Dept. of Information Technology, Internet Based Communication Networks and Services (IBCN) \\ Ghent University - IBBT, Gaston Crommenlaan 8 Bus 201, 9050 Ghent, Belgium \\ Email: \{domenico.spina; francesco.ferranti; tom.dhaene; luc.knockaert\}@intec.ugent.be \\ ${ }^{+}$UAq EMC Laboratory, Dipartimento di Ingegneria Elettrica e dell'Informazione, \\ Università degli Studi dell'Aquila, Via G. Gronchi 18, 67100, L'Aquila, Italy \\ Email: giulio.antonini@univaq.it
}

\begin{abstract}
A novel technique for the variability analysis of generic multiport systems (e.g. interconnections, filters, connectors) is presented. The proposed method describes the statistical properties of a multiport system using the Polynomial Chaos expansion, starting from a set of univariate macromodels of the system transfer function. A numerical example shows the accuracy and efficiency of the proposed approach with respect to standard Monte Carlo analysis.
\end{abstract}

\section{INTRODUCTION}

The increasing demand for performance and miniaturization of the modern integrated circuits have reduced the size and increased the density of these systems, while pushing operation to higher signal bandwidths. Therefore, it is fundamental to estimate the effects of the variability of geometrical and electrical parameters on the integrated circuit performance.

The Monte Carlo (MC) method is the standard approach for variability analysis. It gives accurate results and its implementation is straightforward, but it has a very high computational cost, because the MC method requires a large number of simulations. Over the last years, several techniques were developed to study the stochastic variations of electrical circuits by means of the Polynomial Chaos (PC) expansion [1][4], PC-based methods are much more efficient with respect to the computationally cumbersome MC-based techniques. These PC-based techniques were designed to handle specific systems: multiconductor transmission lines [5], [6] and lumped elements circuits [7].

The proposed method overcomes these limitations and allows to perform variability analysis on a generic multiport system, described by its linear transfer function. In the frequencydomain, the system transfer function can be expressed in different forms (e.g. scattering, impedance or admittance parameters), making the proposed method applicable to a large range of microwave systems.

\section{Polynomial ChaOs CHARACTERISTiCS}

A stochastic process $Y$, under specific conditions [1], can be expressed as a series of orthogonal polynomials as [2]

$$
Y=\sum_{i=0}^{\infty} \alpha_{i} \varphi_{i}(\boldsymbol{\xi})
$$

where $\varphi_{i}(\boldsymbol{\xi})$ are the corresponding orthogonal polynomials, also called basis functions, that depend on the vector of normalized random variables (RVs) $\boldsymbol{\xi}$ and the terms $\alpha_{i}$ are called PC coefficients. The polynomials satisfy the following orthogonality condition [3]

$$
<\varphi_{i}(\boldsymbol{\xi}), \varphi_{j}(\boldsymbol{\xi})>=\int_{\boldsymbol{\Omega}} \varphi_{i}(\boldsymbol{\xi}) \varphi_{j}(\boldsymbol{\xi}) W(\boldsymbol{\xi}) d \boldsymbol{\xi}=a_{i} \delta_{i j}
$$

where $a_{i}$ are positive numbers, $\delta_{i j}$ is the Kronecker delta and $W(\boldsymbol{\xi})$, often referred as weighting function in the theory of orthogonal polynomials [1], is a probability measure with support $\boldsymbol{\Omega}$.

Let us suppose in what follows that the RVs of the stochastic process $Y$ are independent: the global uncertainty probability density function (PDF) is the product of the PDFs of the single $\mathrm{RV}$. In this case, the weighting function is

$$
W(\boldsymbol{\xi})=\prod_{i=1}^{N} W_{i}\left(\xi_{i}\right)
$$

where $N$ is the number of RVs. Therefore, a product combination of the orthogonal polynomials corresponding to each individual RV $\xi_{i}$ can be used to obtain the basis functions $\varphi_{i}(\boldsymbol{\xi})$ [4]. Consequently, it is possible to truncate (1) to a limited number of basic functions $M$ as [2]

$$
M+1=\frac{(N+P) !}{N ! P !}
$$

where $P$ is the highest degree of the polynomials used in the truncated PC expansion.

The basis functions can be calculated numerically for independent RVs with arbitrary PDFs, following the approach described in [3], but for specific PDFs the basis functions are the polynomials of the Wiener-Askey scheme [1]. For example, in the case of RVs with uniform PDFs the basis functions are the Legendre polynomial, while in the Gaussian PDF case the basis functions are the Hermite polynomials. Finally, (1) can be expressed as

$$
Y \approx \sum_{i=0}^{M} \alpha_{i} \varphi_{i}(\boldsymbol{\xi})
$$


where only the $M+1$ PC coefficients $\alpha_{i}$ must be computed.

The PC expansion has an attractive characteristic: the analytical representation of the system variability. Indeed, the mean $\mu$ and the variance $\sigma^{2}$ of the stochastic process $Y$ can be expressed as [2]

$$
\begin{aligned}
\mu & =\alpha_{0} \\
\sigma^{2} & =\sum_{i=1}^{M} \alpha_{i}^{2}<\varphi_{i}(\boldsymbol{\xi}), \varphi_{i}(\boldsymbol{\xi})>
\end{aligned}
$$

Furthermore, following standard analytical formulas or numerical schemes [8], it is possible to calculate also more general stochastic functions of $Y$, such as the PDF.

We note that it is straightforward to express a stochastic process in a matrix form $\boldsymbol{Y}$ by means of the PC expansion: the corresponding PC coefficients must be calculated for each entry of $\boldsymbol{Y}$. Hence, (5) can be recast as

$$
\boldsymbol{Y} \approx \sum_{i=0}^{M} \boldsymbol{\alpha}_{i} \varphi_{i}(\boldsymbol{\xi})
$$

where $\boldsymbol{\alpha}_{i}$ is the matrix of PC coefficients for the $i$-th polynomial basis.

\section{PC MOdEL OF Multiport Systems}

The premise of this work is that the linear transfer function of a multiport system is expressed in a state-space form as

$$
\begin{aligned}
(s \boldsymbol{I}-\boldsymbol{A}(\boldsymbol{\xi})) \boldsymbol{X}(s, \boldsymbol{\xi}) & =\boldsymbol{B}(\boldsymbol{\xi}) \\
\boldsymbol{Y}(s, \boldsymbol{\xi}) & =\boldsymbol{C}(\boldsymbol{\xi}) \boldsymbol{X}(s, \boldsymbol{\xi})+\boldsymbol{D}(\boldsymbol{\xi})
\end{aligned}
$$

where the dependence on a vector of RVs $\boldsymbol{\xi}$ is explicitly indicated. Our goal is to build a PC-model of the form (8) for the output $\boldsymbol{Y}$.

In what follows, we will demonstrate that our goal can be achieved:

- choosing on the number of basis functions $M$;

- computing the PC coefficients of the state-space matrices;

- calculating the corresponding Galerkin projection [7] to determinate the coefficients of the PC expansion of $\boldsymbol{X}$;

- calculating the PC expansion of $\boldsymbol{Y}$ by means of a suitable combination of the results previously obtained.

In our approach, the number of basis functions $M$ is chosen upfront, according to (4). This choice is made considering that $P$ can be limited between two and five [1], [5] for a variability analysis. The linear regression technique [2] is used to compute the PC coefficients. This regression approach allows to calculate all the PC coefficients of the state-space matrices solving a least-square system, calculated with respect to an initial set of discrete samples of the normalized RVs $\left[\boldsymbol{\xi}_{j}\right]_{j=1}^{K}$, of the form [2]

$$
\Psi \alpha=R
$$

The $j$-th row of the matrix $\boldsymbol{\Psi}$ is formed by the multivariate polynomial basis evaluated in $\boldsymbol{\xi}_{j}$, while the matrix $\boldsymbol{R}$ represent the corresponding set of values of the system transfer function.
Hence, the equivalent equation (11) must be built for each state-space matrices in (9), (10). The starting point of the proposed technique is the computation of $K$ univariate frequency-domain macromodels, called root macromodels [9], [10]. This is done by using the Vector Fitting (VF) algorithm [11] $K$ times for a discrete set of values of the normalized RVs $\left[\boldsymbol{\xi}_{j}\right]_{j=1}^{K}$, where the same number of frequency samples is used each time. Afterwards, using a suitable realization technique, a state-space realization is obtained for each root macromodel $\left[\boldsymbol{A}_{j}, \boldsymbol{B}_{j}, \boldsymbol{C}_{j}, \boldsymbol{D}_{j}\right]_{j=1}^{K}$, assuring that all the $K$ realizations of each state-space matrices have common dimensions. The standard Gilbert realization [12] is used in our method.

Finally, an equivalent equation (11) can be obtained for each state-space matrix by ordering in a suitable way the basis functions and the state-space matrices computed for each $\boldsymbol{\xi}_{j}$.

At this point, the state-space matrices are modeled in the form (8), thanks to an a priori estimation of $M$ and the use of the linear regression method. In the following, the PC coefficients of the state-space matrices will be indicated as $\boldsymbol{A}_{i}, \boldsymbol{B}_{i}, \boldsymbol{C}_{i}, \boldsymbol{D}_{i}, i=0, \ldots, M$. Now, let us assume that also the state vector and the output are expressed with a PC model, then (9) and (10) become

$$
\begin{aligned}
s \sum_{j=0}^{M} \boldsymbol{X}_{j}(s) \varphi_{j}(\boldsymbol{\xi}) & =\sum_{i=0}^{M} \sum_{j=0}^{M} \boldsymbol{A}_{i} \boldsymbol{X}_{j}(s) \varphi_{i}(\boldsymbol{\xi}) \varphi_{j}(\boldsymbol{\xi}) \\
& +\sum_{i=0}^{M} \boldsymbol{B}_{i} \varphi_{i}(\boldsymbol{\xi})
\end{aligned}
$$

$$
\begin{aligned}
\sum_{j=0}^{M} \boldsymbol{Y}_{j}(s) \varphi_{j}(\boldsymbol{\xi}) & =\sum_{i=0}^{M} \sum_{j=0}^{M} \boldsymbol{C}_{i} \boldsymbol{X}_{j}(s) \varphi_{i}(\boldsymbol{\xi}) \varphi_{j}(\boldsymbol{\xi}) \\
& +\sum_{i=0}^{M} \boldsymbol{D}_{i} \varphi_{i}(\boldsymbol{\xi})
\end{aligned}
$$

where the only unknown terms are the matrices of PC coefficients $\boldsymbol{X}_{j}(s)$ for the state vector, and $\boldsymbol{Y}_{j}(s)$ for the output.

The desired state-vector coefficients can now be calculated through Galerkin projection [7]. For example, let us assume that the transfer function of the multiport system depends on one RV and $M=2$ basis functions are used for the PC model. These hypotheses are not stringent: it is straightforward to extend the presented formulation to the case of multiple RVs and higher order of expansion. In this simplified case, equation (12) can be rewritten as

$$
\begin{aligned}
s \boldsymbol{X}_{0} \varphi_{0}+s \boldsymbol{X}_{1} \varphi_{1}=\boldsymbol{A}_{0} \boldsymbol{X}_{0} \varphi_{0} \varphi_{0} & \\
+\boldsymbol{A}_{1} \boldsymbol{X}_{0} \varphi_{1} \varphi_{0}+\boldsymbol{A}_{0} \boldsymbol{X}_{1} \varphi_{0} \varphi_{1} & +\boldsymbol{A}_{1} \boldsymbol{X}_{1} \varphi_{1} \varphi_{1} \\
& +\boldsymbol{B}_{0} \varphi_{0}+\boldsymbol{B}_{1} \varphi_{1}
\end{aligned}
$$

where the explicit dependency on the vector $\xi$ is omitted, for the sake of clarity. Projection of (14) onto the basis function 
$\varphi_{p}$ with $p=0,1$, yields

$$
\begin{array}{r}
s \boldsymbol{X}_{0}<\varphi_{0}, \varphi_{p}>+s \boldsymbol{X}_{1}<\varphi_{1}, \varphi_{p}>= \\
\boldsymbol{A}_{0} \boldsymbol{X}_{0}<\varphi_{0} \varphi_{0}, \varphi_{p}>+\boldsymbol{A}_{1} \boldsymbol{X}_{0}<\varphi_{1} \varphi_{0}, \varphi_{p}> \\
+\boldsymbol{A}_{0} \boldsymbol{X}_{1}<\varphi_{0} \varphi_{1}, \varphi_{p}>+\boldsymbol{A}_{1} \boldsymbol{X}_{1}<\varphi_{1} \varphi_{1}, \varphi_{p}> \\
+\boldsymbol{B}_{0}<\varphi_{0}, \varphi_{p}>+\boldsymbol{B}_{1}<\varphi_{1}, \varphi_{p}>
\end{array}
$$

Therefore, the desired matrices $\boldsymbol{X}_{0}$ and $\boldsymbol{X}_{1}$ can be easily calculated once the scalar products in (15) are computed.

Finally, it is straightforward to obtain the PC coefficients of the output $\boldsymbol{Y}_{j}(s)$. Indeed, thanks to the orthogonality relation (2), projecting equation (13) onto the basis function $\varphi_{p}$ with $p=0,1$, leads to

$$
\begin{aligned}
& \boldsymbol{Y}_{p}(s)<\varphi_{p}(\boldsymbol{\xi})>^{2}= \\
& \sum_{i=0}^{1} \sum_{j=0}^{1} \boldsymbol{C}_{i} \boldsymbol{X}_{j}(s)<\varphi_{i}(\boldsymbol{\xi}) \varphi_{j}(\boldsymbol{\xi}), \varphi_{p}(\boldsymbol{\xi})>+\boldsymbol{D}_{p}<\varphi_{p}(\boldsymbol{\xi})>^{2}
\end{aligned}
$$

where all the scalar products in (16) are already known, because they were computed in the previous step to calculate the PC coefficients $\boldsymbol{X}_{j}$ of the state-vector.

\section{NumERICAL EXAMPLE}

A bandpass hairpin filter has been modeled within the frequency range $[1.5-3.5] \mathrm{GHz}$. Its layout is shown in Fig. 1. The substrate is $0.635 \mathrm{~mm}$ thick with a relative dielectric constant $\epsilon_{r}=9.9$. The filter has a length $L=12 \mathrm{~mm}$ and its conductors have a width $W_{1}=0.33 \mathrm{~mm}$, while the conductors at the input or output port have a width of $W_{2}=0.66 \mathrm{~mm}$. The spacing between the conductors at the input or output port and the filter conductors is $S_{1}=S_{2}=0.3 \mathrm{~mm}$ and the spacing between the filter conductors is $D=1 \mathrm{~mm}$. The distance $C$ is equal to $2.5 \mathrm{~mm}$.

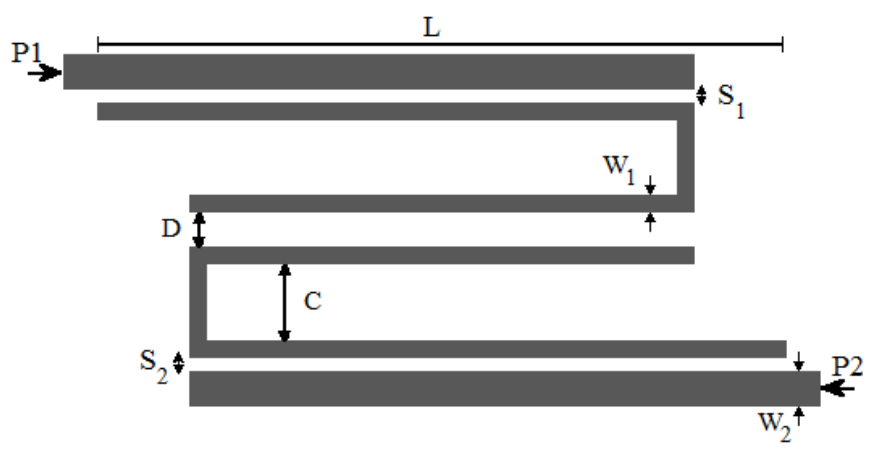

Fig. 1. Layout of the hairpin bandpass filter.

The scattering parameters of the structure are considered as a stochastic process with respect to two independent RVs $(N=2)$ with uniform PDFs: the spacing $S_{1}$ and the distance $D$. Both variables vary in a range of $\pm 10 \%$ with respect to their nominal value. The corresponding basis functions are products of the Legendre polynomials [4], while the weighting function (3) is

$$
W(\boldsymbol{\xi})=\left\{\begin{array}{l}
2^{-N}, \quad\left|\xi_{i}\right| \leq 1, \quad i=1, \ldots, N \\
0, \quad \text { elsewhere }
\end{array}\right.
$$

The scattering parameters are evaluated using ADS Momentum $^{1}$ over a grid composed of 31 samples for the frequency and $6 \times 6\left(S_{1}, D\right)$ samples for the geometrical parameters, while the simulations are performed with MATLAB ${ }^{2} 2010 a$ on a computer with an Intel(R) Core(TM) i3 processor and 4 GB RAM. Note that the scalar products necessary to calculate the Galerkin projection are calculated analytically on beforehand.

The rational models for the initial samples are calculated using the VF algorithm. To estimate the required number of poles, $-60 \mathrm{~dB}$ is chosen as maximum absolute model error between the scattering parameters and the corresponding root macromodels. The PC expansion is calculated using $P=2$ and $M=5$, according to (4). The comparison in Table I illustrates the significant efficiency gain of the proposed technique with respect to the $\mathrm{MC}$ analysis (performed using 10000 samples).

TABLE I

EFFICIENCY OF THE PRoposed PC-BASEd TECHNIQUE

\begin{tabular}{|l|c|}
\hline Technique & Computational time \\
\hline \hline Monte Carlo Analysis (10000 samples) & $208 \mathrm{~h}, 45 \mathrm{~min}, 57.5 \mathrm{~s}$ \\
\hline PC-based technique (36 samples) & $45 \mathrm{~min} 16.6 \mathrm{~s}$ \\
\hline Details PC-based technique & Computational time \\
\hline Initial simulations & $45 \mathrm{~min} 5.6 \mathrm{~s}$ \\
\hline PC model scattering parameters & $11 \mathrm{~s}$ \\
\hline
\end{tabular}

Fig. 2 shows the results of the MC analysis for the magnitude of $S_{11}$. It is worth noticing that, for the central frequency $2.5 \mathrm{GHz}$, the filter has a high variability with respect to the chosen RVs. Figs. 3, 4 show an example of the accuracy of the proposed PC-based technique with respect to the classical $\mathrm{MC}$ analysis, in computing the first two stochastic moments of the scattering parameters. Furthermore, the PC-based model is able to accurately represent also the PDF of the process, as shown in Fig. 5 for the filter central frequency. Similar results can be obtained for all other entries of the scattering matrix.

\section{CONClusions}

We have presented a novel technique for efficient variability analysis of general multiport systems. The system variability features are described using the PC expansion applied to rational root macromodels of the system transfer function. The proposed approach presents a high degree of versatility,

\footnotetext{
${ }^{1}$ Momentum EEsof EDA, Agilent Technologies, Santa Rosa, CA.

${ }^{2}$ The Mathworks, Inc., Natick
} 


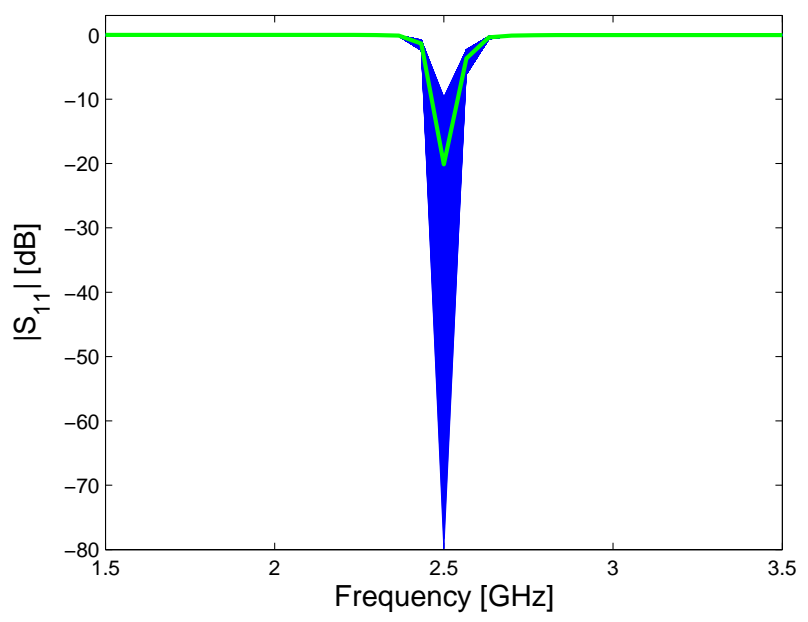

Fig. 2. Variability of the magnitude of $S_{11}$. The green thick line corresponds to the nominal value for $S_{1}$ and $D$.

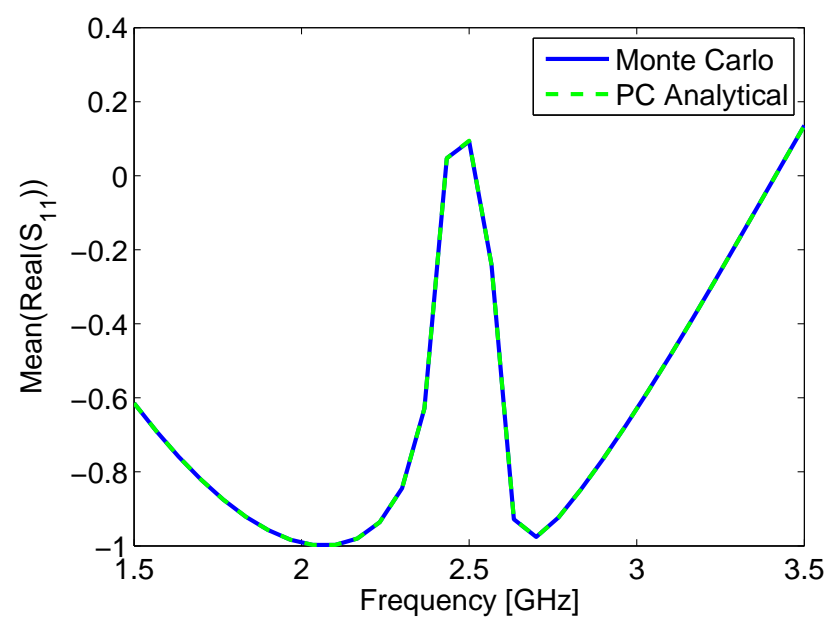

Fig. 3. Mean for the real part of $S_{11}$ obtained with the MC analysis and the proposed PC-based method.

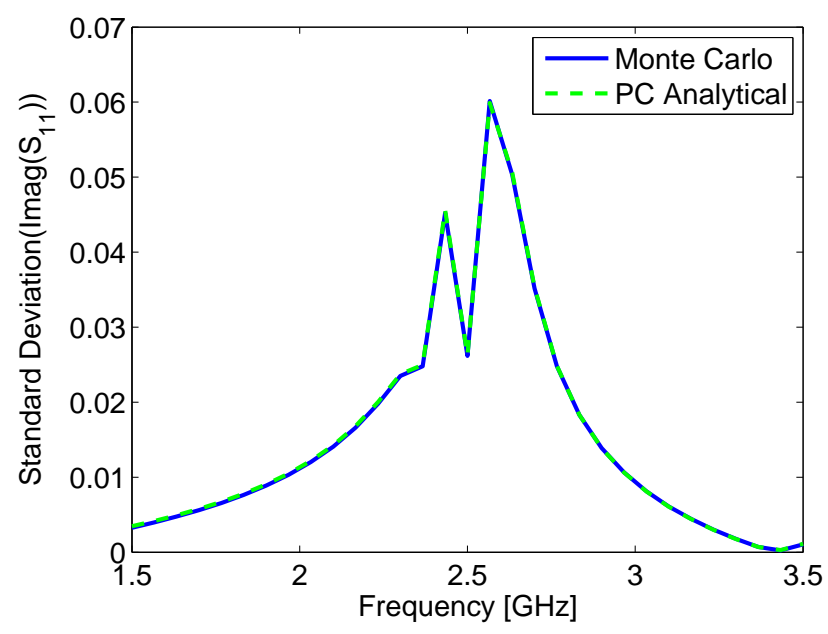

Fig. 4. Standard deviation for the imaginary part of $S_{11}$ obtained with the MC analysis and the proposed PC-based method.

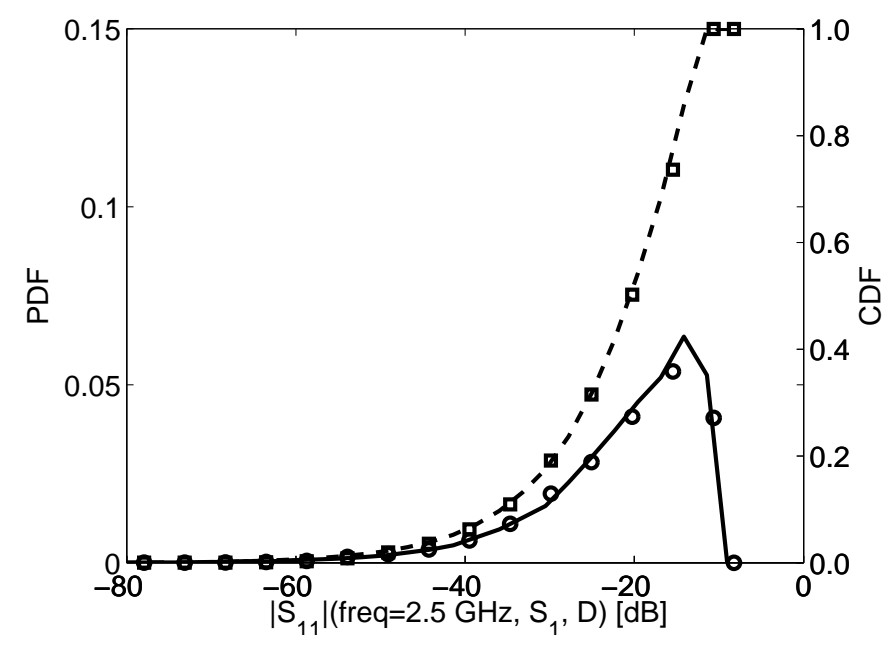

Fig. 5. PDF and CDF of the magnitude of $S_{11}$ at $2.5 \mathrm{GHz}$. Full black line: PDF computed using the novel technique; Dashed black line: CDF computed using the novel technique; Circles (o): PDF computed using the MC technique; Squares ( $\square)$ : CDF computed using the MC technique.

allowing to express the system transfer function in different forms (e.g. scattering, impedance or admittance parameters). Comparison results with the standard MC approach validate the accuracy and computational efficiency of the new proposed method.

\section{REFERENCES}

[1] D. Xiu and G.M. Karniadakis, "The Wiener-Askey polynomial chaos for stochastic differential equations," SIAM J. Sci. Comput., vol. 24, no. 2, pp. 619-644, Apr. 2002.

[2] M. S. Eldred, "Recent advance in non-intrusive polynomial-chaos and stochastic collocation methods for uncertainty analysis and design," in Proc. 50th AIAA/ASME/ASCE/AHS/ASC Structures, Structural Dynamics, and Materials Conference, Palm Springs, California, May 2009.

[3] J. A. S. Witteveen and H. Bijl, "Modeling Arbitrary Uncertainties Using Gram-Schmidt Polynomial Chaos," in Proc. 44th AIAA Aerospace Sciences Meeting and Exhibit, no. AIAA-2006-0896, Palm Springs, California, Jan. 2006.

[4] C. Soize and R. Ghanem, "Physical systems with random uncertainties: Chaos representations with arbitrary probability measure," SIAM J. SCI. COMPUT., vol. 26, no. 2, pp. 395-410, Jul. 2004.

[5] I. S. Stievano, P. Manfredi, F. G. Canavero, "Parameters variability effects on multiconductor interconnects via Hermite polynomial chaos," IEEE Trans. Compon., Packag., Manuf. Technol., vol. 1, no. 8, pp. 12341239, 2011.

[6] - "Stochastic analysis of multiconductor cables and interconnects," IEEE Trans. Electromagn. Compat., vol. 53, no. 2, pp. 501-507, 2011.

[7] Q. Su and K. Strunz, "Stochastic polynomial-chaos-based average modeling of power electronic systems," Power Electronics, IEEE Transactions on, vol. 26, no. 4, pp. $1167-1171$, Apr. 2011.

[8] A. Papoulis, Probability, Random Variables and Stochastic Processes. Mcgraw-Hill College, 1991.

[9] F. Ferranti, L. Knockaert and T. Dhaene, "Parameterized S-parameter based macromodeling with guaranteed passivity," IEEE Microw. Wireless Compon. Lett., vol. 19, no. 10, pp. 608-610, 2009.

[10] F. Ferranti, L. Knockaert, T. Dhaene and Giulio Antonini,, "Passivitypreserving parametric macromodeling for highly dynamic tabulated data based on Lur'e equations," IEEE Trans. Microw. Theory Tech., vol. 58, no. 12 , pp. 3688-3696, 2010.

[11] B. Gustavsen and A. Semlyen, "Rational approximation of frequency domain responses by vector fitting," IEEE Trans. Power Del., vol. 14, no. 3, pp. 1052-1061, Jul. 1999.

[12] E. G. Gilbert, "Controllability and observability in multivariable control systems," SIAM Journal on Control, vol. 2, no. 1, pp. 128-151, 1963. 